\title{
SURFACES IN $\mathbb{P}^{4}$ FIBERED IN CUBICS
}

\author{
ALBERTO ALZATI AND ALFONSO TORTORA
}

\begin{abstract}
Any algebraic surface in $\mathbb{P}^{n}(\mathbb{C})$ which is fibered in cubics, such that the generic fibre is a twisted cubic, gives rise to a curve $\Gamma$ in a suitable compactification $X$ of the space of smooth rational cubics of $\mathbb{P}^{n}(\mathbb{C})$. In this paper the case $n=4$ is addressed and the corresponding space $X$ is studied. We apply our results to complete the classification of smooth, rational, surfaces in $\mathbb{P}^{4}(\mathbb{C})$ ruled in cubics.
\end{abstract}

\section{INTRODUCTION}

Let $S$ be a smooth, nondgenerate, surface embedded in $\mathbb{P}^{4}(\mathbb{C})$. Let us assume that there exists a morphism $\pi: S \rightarrow C$, where $C$ is a smooth curve, such that the generic fibre of $\pi$ is embedded as a smooth twisted cubic and the special ones are embedded as reducible cubics. Under these assumptions $S$ can be considered as a curve $\Gamma$ in a suitable compactification $X$ of the space of smooth rational cubics of $\mathbb{P}^{4}(\mathbb{C})$. On the other hand, a generic projection of $S$ in $\mathbb{P}^{2}(\mathbb{C})$ gives rise to a one dimensional family of plane cubic curves whose general member is a nodal cubic. The knowledge of the Chow ring of $X$ and the well known enumerative geometry of nodal plane cubics (see $[\mathrm{K}-\mathrm{S}]$ ) allow us to give some costraints to the number and the type of singular fibres. In some cases these costraints can be used to exclude the existence of $S$.

In this short note we apply the above program to 5 types of surfaces $S$ whose existence was left as an open problem in [E]. More precisely: in $\S 2$ we study the structure of $X$ and in particular its Chow ring. In $\S 3$, by using the results of $\S 2$, we solve the problem, see theorem 3.1. In $\S 4$ we consider the problem of classifying of all $\mathbb{P}^{1}$-bundles in $\mathbb{P}^{4}(\mathbb{C})$ when $\operatorname{deg}(S) \geq 16$, since, by the well known conjecture about smooth surfaces of non general type in $\mathbb{P}^{4}(\mathbb{C})$, when $\operatorname{deg}(S) \leq 15$ there is only one possible surface of this type. We reduce the problem to consider only two possible cases in which all fibres are embedded as smooth rational cubics. Unfortunately the above program does not work in these cases.

Acknowledgements: we would like to thank S. A. Stromme for some helpful conversations on the subject of $\S 2$.

Date: January 30, 2009.

1991 Mathematics Subject Classification. Primary 14M07, 14J26.

Key words and phrases. ruled surfaces, low codimension varieties.

This work is within the framework of the national research project "Geomety on Algebraic Varieties" Cofin 2006 of MIUR. 


\section{The SPACE OF TWISTED CUBICs IN $\mathrm{P}^{4}$}

First of all we want to prove the following

Theorem 2.1. There exists a minimal compactification $X$ of the space of twisted cubics in $\mathbb{P}^{4}(\mathbb{C})$ such that:

i) $X$ is a smooth projective variety of dimension 16

ii) $X$ is a fibered space over $\mathbb{P}^{4}(\mathbb{C})^{*}$ and every fibre is isomorphic to a suitable compactification of the space of twisted cubics in $\mathbb{P}^{3}(\mathbb{C})$

iii) $A^{1}(X) \otimes \mathbb{Q}$ is generated by two elements, say $\eta$ and $\tau$, where $\eta$ is the pull-back of the generator of $A^{1}\left(\mathbb{P}^{4}(\mathbb{C})^{*}\right)$ and $\tau$ is the complement of the set of twisted cubics in $\mathbb{P}^{4}(\mathbb{C})$.

Note that $i$ ) and $i$ ) are in fact proved in [E-S]. However, since the description of $A^{1}(X) \otimes \mathbb{Q}$ given there does not suit our purpose, we prefer to present a proof of $i$ ) and $i i$ ) in order to obtain $i i i)$.

In what follows we use the ideas of [E-P-S], where the authors give a compactification for the space of twisted cubics in $\mathbb{P}^{3}(\mathbb{C})$ by using the fact that every twisted cubic is the locus in $\mathbb{P}^{3}(\mathbb{C})$ where a $(2,3)$ matrix of linear forms drops rank. Hence they consider the set of such matrices, suitably identified by the action of some group. Here we consider $(2,3)$ matrices of linear forms in $\mathbb{P}^{4}(\mathbb{C})$ suitably identified by the action of an analogous group and by an equivalence relation given by the restriction to hyperplanes of $\mathbb{P}^{4}(\mathbb{C})$. Before giving the proof of theorem 2.1 we need some background and some lemmas.

Let $E$ and $F$ be two vector spaces over $\mathbb{C}$ of dimension 3 and 2 respectively. Let $V$ be a 5 -dimensional vector space over $\mathbb{C}$ such that $\mathbb{P}^{4}(\mathbb{C})=\mathbb{P}\left(V^{*}\right)$. Let us define $W_{1}:=\operatorname{Hom}(F, E \otimes V) \times(V \backslash\{\mathbf{0}\})$. Any element of $W_{1}$ is a pair $(f, \mathbf{v})$ and, if we fix bases in $F, E, V, f$ and $\mathbf{v}$ correspond to two matrices $A$ and $\mathbf{b}$. $A$ is a $(3,2)$ matrix of linear forms in $\mathbb{P}^{4}(\mathbb{C})$, b is a $(5,1)$ non zero vector of $\mathbb{C}^{5}$.

Let us introduce in $W_{1}$ the following equivalence relation: $(f, \mathbf{v}) \sim\left(f^{\prime}, \mathbf{v}\right)$ if and only if $\left\langle\mathbf{v}^{\prime}\right\rangle=\langle\mathbf{v}\rangle$ and $\left.f^{\prime}-f \in \operatorname{Hom}(F, E \otimes<\mathbf{v}\rangle\right)$. We define $W:=W_{1} / \sim$ and, by abuse of notation, we denote the corresponding equivalence class by the same symbol $(f, \mathbf{v})$.

Let $G_{1}$ be the group $G L(F) \times G L(E) \times \operatorname{Hom}(F, E)$ where the product of two elements is defined by:

$$
(\alpha, \beta, \gamma) \cdot\left(\alpha^{\prime}, \beta^{\prime}, \gamma^{\prime}\right):=\left(\alpha \circ \alpha^{\prime}, \beta \circ \beta^{\prime}, \gamma^{\prime}+\beta^{\prime-1} \circ \gamma \circ \alpha^{\prime}\right)
$$

$G_{1}$ is a group indeed, whose unity is $\left(i d_{F}, i d_{E}, 0\right)$ and it acts on $W$ in the following way:

$$
(\alpha, \beta, \gamma) \cdot(f, \mathbf{v}):=\left(\left(\beta \otimes i d_{V}\right) \circ f \circ \alpha^{-1}+\left(\beta \circ \gamma \circ \alpha^{-1}\right)_{\mathbf{v}}, \mathbf{v}\right)
$$

where $\left(\beta \circ \gamma \circ \alpha^{-1}\right)_{\mathbf{v}}(\mathbf{w}):=\left(\beta \circ \gamma \circ \alpha^{-1}\right)(\mathbf{w}) \otimes \mathbf{v}$ for any $\mathbf{w} \in F$.

Let $\Gamma$ be the normal subgroup $\left\{a \cdot i d_{E}, a \cdot i d_{F}, \gamma\right\} a \in \mathbb{C}^{*}, \gamma \in \operatorname{Hom}(F, E)$, of $G_{1}$. It is easy to see that $\Gamma$ acts trivially on $W$, hence the group $G:=G_{1} / \Gamma$ acts on $W$. Note that $W$ is a fiber space over $\mathbb{P}^{4}(\mathbb{C})^{*}$ whose fibration is given by the natural $\operatorname{map} p: W \rightarrow \mathbb{P}^{4}(\mathbb{C})^{*}$ such that $p(f, \mathbf{v}):=<\mathbf{v}>$. Moreover $p$ is $G$-invariant.

For any $\mathbf{v} \neq \mathbf{0}$ let $\pi_{\mathbf{v}}$ be the natural projection $V \rightarrow V_{/<\mathbf{v}>}$. For any $(f, \mathbf{v}) \in W$, (recall that $\mathbf{v} \neq \mathbf{0})$, we define $\phi_{\mathbf{v}}(f):=\left(i d_{E} \otimes \pi_{\mathbf{v}}\right) \circ f \in \operatorname{Hom}\left(F, E \otimes V_{/<\mathbf{v}>}\right)$. If we fix bases for $E, F, V$, we can identify $\phi_{\mathbf{v}}(f)$ with a $(3,2)$ matrix of linear forms on the hyperplane $\mathbb{P}\left(\left(V_{/<\mathbf{v}>}\right)^{*}\right) \simeq \mathbb{P}^{3}(\mathbb{C})$ of $\mathbb{P}^{4}(\mathbb{C})=\mathbb{P}\left(V^{*}\right)$. By taking the $(2,2)$ minors of this matrix, we get a linear system of quadrics $Q_{(f, \mathbf{v})}$ in $\mathbb{P}\left(\left(V_{/<\mathbf{v}>}\right)^{*}\right)$. 
Let $U:=\left\{(f, \mathbf{v}) \in W \mid \operatorname{dim}\left(Q_{(f, \mathbf{v})}\right)=3\right\}$. Let $\mathcal{G}$ be the Grassmannian bundle over $\mathbb{P}^{4}(\mathbb{C})^{*}=\mathbb{P}(V)$, whose fibre over $\mathbf{v}$ is $G\left(3, S^{2}\left(V_{/<\mathbf{v}>}\right)\right)$, the Grassmannian of 3dimensional linear subspace of $S^{2}\left(V_{/<\mathbf{v}>}\right)$, then we can define a map $\Phi: U \rightarrow \mathcal{G}$ by $\Phi(f, \mathbf{v})=Q_{(f, \mathbf{v})}$. Let $X$ be $\operatorname{Im}(\Phi)$. Note that this definition coincides with the definition of $\mathcal{X}$ given in [E-S], page 12 .

The group $G_{E P S}:=G L(F) \times G L(E) /\left[\left\{a \cdot i d_{F}, a \cdot i d_{E}\right\} a \in \mathbb{C}^{*}\right]$ acts in a natural way on $\operatorname{Hom}\left(F, E \otimes V_{\mid<\mathbf{v}>}\right)$ as explained in [E-P-S]. Note that $G$ is isomorphic to $G_{E P S}$ via the natural projection $G_{1} \rightarrow G_{E P S}$ whose kernel is $\Gamma$. Concerning $G_{E P S}$ we have the following simple

Lemma 2.2. Two elements $(f, \mathbf{v}) \in W$ and $\left(f^{\prime}, \mathbf{v}\right) \in W$ are $G$-equivalent if and only if $\phi_{\mathbf{v}}(f)$ and $\phi_{\mathbf{v}}\left(f^{\prime}\right)$ are $G_{E P S}$-equivalent.

Proof. Assume that $\phi_{\mathbf{v}}(f)$ and $\phi_{\mathbf{v}}\left(f^{\prime}\right)$ are $G_{E P S}$-equivalent. Then there exist $(\alpha, \beta) \in G L(E) \times G L(F)$ such that

$$
\left(i d_{E} \otimes \pi_{\mathbf{v}}\right) \circ f^{\prime}=\left(\beta \otimes i d_{V_{/<\mathbf{v}>}}\right) \circ\left(i d_{E} \otimes \pi_{\mathbf{v}}\right) \circ f \circ \alpha^{-1} .
$$

$\operatorname{But}\left(\beta \otimes i d_{\left.V_{/<\mathbf{v}}\right)}\right) \circ\left(i d_{E} \otimes \pi_{\mathbf{v}}\right)=\left(i d_{E} \otimes \pi_{\mathbf{v}}\right) \circ\left(\beta \otimes i d_{V}\right)$, so that we get:

$$
\left(i d_{E} \otimes \pi_{\mathbf{v}}\right) \circ\left[f^{\prime}-\left(\beta \otimes i d_{V}\right) \circ f \circ \alpha^{-1}\right]=0
$$

i.e. $f^{\prime}-\left(\beta \otimes i d_{V}\right) \circ f \circ \alpha^{-1} \in \operatorname{Hom}(F, E \otimes<\mathbf{v}>)$. Then there exists a suitable $\gamma \in \operatorname{Hom}(F, E)$ such that

$$
f^{\prime}-\left(\beta \otimes i d_{V}\right) \circ f \circ \alpha^{-1}=\left(\beta \circ \gamma \circ \alpha^{-1}\right)_{\mathbf{v}}
$$

i.e. $(f, \mathbf{v}) \in W$ and $\left(f^{\prime}, \mathbf{v}\right) \in W$ are $G$-equivalent.

The converse is proved in the same way.

It follows from lemma 2.2 that, for any $u, u^{\prime} \in U, \Phi(u)=\Phi\left(u^{\prime}\right)$ if and only if $u$ and $u^{\prime}$ are $G$-equivalent, so $X \simeq U_{/ G}$ as sets. In fact if we fix any $\mathbf{v} \neq \mathbf{0}$, and $u=(f, \mathbf{v})$ and $u^{\prime}=\left(f^{\prime}, \mathbf{v}\right)$ are such that $\Phi(u)=\Phi\left(u^{\prime}\right)$, then $Q_{(f, \mathbf{v})}$ and $Q_{\left(f^{\prime}, \mathbf{v}\right)}$ are the same net in $\mathbb{P}\left(\left(V_{\mid<\mathbf{v}>}\right)^{*}\right)$, hence they are $G_{E P S}$-equivalent and then $u$ and $u^{\prime}$ are $G$-equivalent by lemma 2.2. For the converse we can argue in the same way.

Now we study the fibres of $p$. For any $\mathbf{v} \neq \mathbf{0}$, the fibre $W_{\mathbf{v}}:=p^{-1}(<\mathbf{v}>)$ is a vector space of dimension 24 isomorphic to $\operatorname{Hom}(F, E \otimes V) / \operatorname{Hom}(F, E \otimes<\mathbf{v}>)$. As we have already seen, $G$ acts also on $W_{\mathbf{v}}$ and we have the following:

Lemma 2.3. Any $(f, \mathbf{v}) \in W_{\mathbf{v}}$ is semistable under the action of $G$ if and only if it is stable under the action of $G$ if and only if it belongs to $U_{\mathbf{v}}:=U \cap W_{\mathbf{v}}$ Moreover any element $(f, \mathbf{v}) \in U^{c}$ can be $G$-equivalent only to an element belonging to $U^{c}$.

Proof. For technical reasons it is useful to consider $P:=\mathbb{P}\left(W_{\mathbf{v}}\right)$ and the group $S:=S L(F) \times S L(E)$, acting on $P$ with the obvious action induced by $G$. For any non zero vector $\mathbf{w} \in W_{\mathbf{v}}$ we denote by $[\mathbf{w}]$ the corresponding point of $P$. As any element of $U_{\mathbf{v}}$ is different from the zero of $W_{\mathbf{v}}$ we can consider $\left[U_{\mathbf{v}}\right]:=\mathbb{P}\left(U_{\mathbf{v}}\right) \subset P$ and our goal become to prove that a point of $P$ is semistable under the action of $S$ if and only if it is stable under the action of $S$ if and only if it belongs to $\left[U_{\mathbf{v}}\right]$. The advantage to work with projective spaces is the possibility to use some useful criteria of stability. For any $\mathbf{v} \neq \mathbf{0}$ we can define $P_{\mathbf{v}}:=\mathbb{P}\left[\operatorname{Hom}\left(F, E \otimes V_{/<\mathbf{v}>}\right)\right]$ and the group $S$ acts on $P_{\mathbf{v}}$ as in [E-P-S]. We can change the proof of lemma 2.2 to get that two points $[f, \mathbf{v}],\left[f^{\prime}, \mathbf{v}\right] \in P$ are $S$-equivalent if and only if $\left[\phi_{\mathbf{v}}(f)\right]$, $\left[\phi_{\mathbf{v}}\left(f^{\prime}\right)\right] \in P_{\mathbf{v}}$ are $S$-equivalent. Note that any point in $P_{\mathbf{v}}$ is $\left[\phi_{\mathbf{v}}(f)\right]$ for at least 
a point $[f, \mathbf{v}] \in P$. Moreover to any point $[f, \mathbf{v}] \in P$ we can associate a unique point $\left[\phi_{\mathbf{v}}(f)\right] \in P_{\mathbf{v}}$. In fact, $\forall a \in \mathbb{C}^{*},[a f, a \mathbf{v}] \rightarrow\left[\phi_{a \mathbf{v}}(a f)\right]=\left[\left(i d_{E} \otimes \pi_{a \mathbf{v}}\right) \circ a f\right]=$ $\left[\left(i d_{E} \otimes \pi_{\mathbf{v}}\right) \circ a f\right]=\left[a\left(i d_{E} \otimes \pi_{\mathbf{v}}\right) \circ f\right]=\left[\left(i d_{E} \otimes \pi_{\mathbf{v}}\right) \circ f\right]=\left[\phi_{\mathbf{v}}(f)\right]$.

Fixing bases for $F, E, V$ it is easy to see that the action of $S$ is in fact a linear action on $\mathbb{P}^{23}(\mathbb{C})$. Moreover $S$ is a reductive group so that we have that a point $[f, \mathbf{v}] \in P$ is semistable if and only if there exists a $S$-invariant homogeneous polynomial $\psi$ in $\mathbb{C}\left[x_{0}, \ldots, x_{23}\right]$, of positive degree, such that $\psi([f, \mathbf{v}]) \neq 0$ (see $[\mathrm{N}]$ pag. $73)$.

For any $\psi$ as above and any $\mathbf{v} \neq \mathbf{0}$ we can define an induced homogeneous polynomial $\psi_{\mathbf{v}}$, of the same degree, which is a function on $P_{\mathbf{v}}$ in this way:

$\psi_{\mathbf{v}}\left(\left[\phi_{\mathbf{v}}(f)\right]\right):=\psi([f, \mathbf{v}])$.

The definition is well-posed. In fact, if $\left[f^{\prime}, \mathbf{v}^{\prime}\right]$ is such that $\left[\phi_{\mathbf{v}}(f)\right]=\left[\phi_{\mathbf{v}^{\prime}}\left(f^{\prime}\right)\right]$ then there exists a suitable $a \in \mathbb{C}^{*}$ such that $\phi_{\mathbf{v}^{\prime}}\left(f^{\prime}\right)=a \phi_{\mathbf{v}}(f)$, i.e. $\left(i d_{E} \otimes \pi_{\mathbf{v}}\right) \circ f^{\prime}=$ $a\left(i d_{E} \otimes \pi_{\mathbf{v}}\right) \circ f$, i.e. $\left(i d_{E} \otimes \pi_{\mathbf{v}}\right) \circ f^{\prime}=\left(i d_{E} \otimes \pi_{\mathbf{v}}\right) \circ(a f)$. But this is possible if and only if there exist suitable $b \in \mathbb{C}^{*}$ and $\varphi \in \operatorname{Hom}(F, E)$ such that: $f^{\prime}=a f+\varphi_{\mathbf{v}}, \mathbf{v}^{\prime}=b \mathbf{v}$. Hence $\left[f^{\prime}, \mathbf{v}^{\prime}\right]=\left[a f+\varphi_{\mathbf{v}}, b \mathbf{v}\right]=\left[\frac{a}{b} f+\frac{1}{b} \varphi_{\mathbf{v}}, \mathbf{v}\right]$ (recall that $\left[\phi_{\theta \mathbf{v}}(\theta f)\right]=\left[\phi_{\mathbf{v}}(f)\right]$ for any $\left.\theta \in \mathbb{C}^{*}\right)$ and it is easy to see that $(f, \mathbf{v})$ and $\left(\frac{a}{b} f+\frac{1}{b} \varphi_{\mathbf{v}}, \mathbf{v}\right)$ are $G$-equivalent, hence $[f, \mathbf{v}]$ and $\left[\frac{a}{b} f+\frac{1}{b} \varphi_{\mathbf{v}}, \mathbf{v}\right]$ are $S$-equivalent and $\psi([f, \mathbf{v}])=\psi\left(\left[\frac{a}{b} f+\frac{1}{b} \varphi_{\mathbf{v}}, \mathbf{v}\right]\right)$. Note also that $\psi_{\mathbf{v}}$ is $S$-invariant by the above version of lemma 2.2 .

If $[f, \mathbf{v}] \in P$ is a semistable point then there exists an $S$-invariant homogeneous polynomial $\psi$ in $\mathbb{C}\left[x_{0}, \ldots, x_{23}\right]$, of positive degree, such that $\psi([f, \mathbf{v}]) \neq 0$. Then $\psi_{\mathbf{v}}$ is an $S$-invariant homogeneous polynomial in $\mathbb{C}\left[y_{0}, \ldots, y_{23}\right]$, of positive degree, such that $\psi_{\mathbf{v}}\left(\left[\phi_{\mathbf{v}}(f)\right]\right) \neq 0$. Hence $\left[\phi_{\mathbf{v}}(f)\right]$ is a semistable point in $P_{\mathbf{v}}$ under the action of $S$ and therefore $\operatorname{dim}\left(Q_{(f, \mathbf{v})}\right)=3$ and $[f, \mathbf{v}] \in\left[U_{\mathbf{v}}\right]$ by lemma 1 of [E-P-S].

Conversely let $[f, \mathbf{v}] \in\left[U_{\mathbf{v}}\right]$ and let us assume that $[f, \mathbf{v}]$ is not semistable under the action of $S$. Then there exists a suitable 1-parameter subgroup $\Sigma \subset S$, acting on $P$, such that $\mu([f, \mathbf{v}], \Sigma)<0$ (see $[\mathrm{N}]$, pag 104-105). As $S$ acts on $P_{\mathbf{v}}$ too, we get a 1-parameter subgroup $\Sigma$ acting on $P_{\mathbf{v}}$ and $\mu\left(\left[\phi_{\mathbf{v}}(f)\right], \Sigma\right)=\mu([f, \mathbf{v}], \Sigma)<0$ by the definition of $\mu$, hence $\left[\phi_{\mathbf{v}}(f)\right]$ is not semistable in $P_{\mathbf{v}}$ under the action of $S$. By lemma 1 of $[$ E-P-S $]$ this is possible only if $\operatorname{dim}\left(Q_{(f, \mathbf{v})}\right) \leq 2$, i.e. if $[f, \mathbf{v}] \notin\left[U_{\mathbf{v}}\right]$ : contradiction.

Now let us prove that a point $[f, \mathbf{v}] \in P$ is stable under the action of $S$ if and only if it belongs to $\left[U_{\mathbf{v}}\right]$. Let $[f, \mathbf{v}]$ be a stable point in $P$ under the action of $S$. Obviously $[f, \mathbf{v}]$ is also a semistable point in $P$ under the action of $S$, hence it is contained in $\left[U_{\mathbf{v}}\right]$ by the previous proof regarding semistable points of $P$. Viceversa, let us consider a point $[f, \mathbf{v}] \in\left[U_{\mathbf{v}}\right]$ and let us assume that it is not stable. Then there exists a suitable 1-parameter subgroup $\Sigma \subset S$, acting on $P$, such that $\mu([f, \mathbf{v}], \Sigma) \leq 0$ (see $[\mathrm{N}]$, pag 104-105). By arguing as before this is possible only if $\operatorname{dim}\left(Q_{(f, \mathbf{v})}\right) \leq 2$, i.e. if $[f, \mathbf{v}] \notin\left[U_{\mathbf{v}}\right]$ : contradiction.

For the last part of the proof we return to $W_{\mathbf{v}}$. If there were two $G$-equivalent elements $(f, \mathbf{v}) \in U^{c}$ and $\left(f^{\prime}, \mathbf{v}\right) \in U$, then, by lemma 2.2, $\phi_{\mathbf{v}}(f)$ and $\phi_{\mathbf{v}}\left(f^{\prime}\right)$ would be $G_{E P S}$-equivalent. By lemma 2 of [E-P-S] this is possible if and only if $\operatorname{dim}\left[Q_{(f, \mathbf{v})}\right]=\operatorname{dim}\left[Q_{\left(f^{\prime}, \mathbf{v}\right)}\right]=2:$ contradiction.

Lemma 2.4. For any $(f, \mathbf{v}) \in U$ let $\mathcal{I}_{Q_{(f, \mathbf{v})}}$ be the ideal generated by the quadrics of $Q_{(f, \mathbf{v})}$ in $\mathbb{P}\left(\left(V_{/<\mathbf{v}>}\right)^{*}\right) \simeq \mathbb{P}^{3}(\mathbb{C})$. Let $Z \subseteq U$ be the set of elements $(f, \mathbf{v}) \in U$ such that $V\left(\mathcal{I}_{Q_{(f, \mathbf{v})}}\right)$ is not a curve. Then any two elements $(f, \mathbf{v})$ and $\left(f^{\prime}, \mathbf{v}\right) \in Z$ are $G$-equivalent and none of the elements $(f, \mathbf{v}) \in Z^{c}$ can be $G$-equivalent to any $\left(f^{\prime}, \mathbf{v}\right) \in Z$. 
Proof. Let $(f, \mathbf{v})$ and $\left(f^{\prime}, \mathbf{v}\right) \in Z$. By lemma 3 of $\left[\right.$ E-P-S] we know that $\phi_{\mathbf{v}}(f)$ and $\phi_{\mathbf{v}}\left(f^{\prime}\right)$ are $G_{E P S}$-equivalent, then they are $G$-equivalent by the lemma 2.2. Viceversa we can argue as in the second part of the proof of lemma 2.3.

We have the following last:

Lemma 2.5. For any $u=(f, \mathbf{v}) \in U$ the derivative $d_{u} \Phi$ at $u$ has rank 16 .

Proof. Let us fix an element $u=(f, \mathbf{v}) \in U$ and let us consider the linear map $d_{u}$ induced by $\Phi$ from the tangent space of $U$ at $u=(f, \mathbf{v})$, which is isomorphic to $W_{1}$, and the tangent space to $X$ at $\Phi(u)$, which is isomorphic to

$\operatorname{Hom}\left(Q_{(f, \mathbf{v})}, S^{2}\left(V_{/<\mathbf{v}>}\right) / Q_{(f, \mathbf{v})}\right) \oplus T_{\mathbf{v}^{*}} \mathbb{P}\left(V^{*}\right)$.

Let $p_{\mathbf{v}}: \operatorname{Hom}(F, E \otimes V) \rightarrow \operatorname{Hom}\left(F, E \otimes V_{/<\mathbf{v}>}\right)$ be the natural linear surjective affine projection.

Let $\delta: V \rightarrow V^{*}$ be a fixed isomorphism between $V$ and $V^{*}$. Then $d_{u}$ is the composition of $p_{\mathbf{v}} \times \delta: H o m(F, E \otimes V) \times V \rightarrow H o m\left(F, E \otimes V_{/<\mathbf{v}>}\right) \times V^{*}$ with $\psi_{E P S} \times P_{V^{*}}: \operatorname{Hom}\left(F, E \otimes V_{/<\mathbf{v}>}\right) \times V^{*} \rightarrow \operatorname{Hom}\left(Q_{(f, \mathbf{v})}, S^{2}\left(V_{/<\mathbf{v}>}\right) / Q_{(f, \mathbf{v})}\right) \oplus T_{\mathbf{v}^{*}} \mathbb{P}\left(V^{*}\right)$ where $\psi_{E P S}$ is the linear map defined in [E-P-S] and $P_{V^{*}}$ is the natural map between a vector space and its projectivization. By lemma 4 of [E-P-S] we know that the rank of $\psi_{E P S}$ is 12 , and the rank of $P_{V^{*}}$ is 4 , then we are done.

Now we can prove theorem 2.1.

Proof. (of theorem 2.1) By the above lemmas and G.I.T. (see th.3.14 of [N]) we can conclude that $U_{\mathbf{v} / G}$ is a smooth projective variety of dimension 12 and by the identification $X \simeq U_{/ G}$ we have that the same facts are true for the fibres of the map $X \rightarrow \mathbb{P}^{4}(\mathbb{C})^{*}$. I.e. we have $i$ ) and $i i$ ) of 2.1 , but, moreover, we also have that in every fibre, according to [E-P-S], there is a divisor, say $\tau_{v}$, whose complement is given by the twisted cubics of $\mathbb{P}\left[\left(V_{/<\mathbf{v}>}\right)^{*}\right]$. In what follows we will show that there exists a divisor $\tau$ in $X$ such that its complement is given by the twisted cubics of $\mathbb{P}^{4}(\mathbb{C})$. In fact the construction of $\tau$ is analogous to the construction of $\tau_{v}$ in [E-P-S] so that the restriction of $\tau$ to any fibre is $\tau_{v}$.

The group $G$ acts freely on $U$. In fact, let us assume that there exists an element $(f, \mathbf{v}) \in U$ and an element $(\alpha, \beta, \gamma) \in G$ such that $(\alpha, \beta, \gamma)(f, \mathbf{v})=(f, \mathbf{v})$. Then $\phi_{\mathbf{v}}(f)$ is a fixed element in $\operatorname{Hom}\left(F, E \otimes V_{/<\mathbf{v}>}\right)$ under the action of the element $(\alpha, \beta)$ of $G_{E P S}$ by lemma 2.2. We know that this implies $(\alpha, \beta) \in\left[\left\{a \cdot i d_{F}, a \cdot i d_{E}\right\}\right.$ $a \in \mathbb{C}^{*}$ ] (see [E-P-S] pag. 89-90) hence we have that $(\alpha, \beta, \gamma) \in \Gamma$. Therefore $U$ is a principal homogeneous $G$-bundle (see [F-M], prop. 0.9)

Now we want to determine the 1-codimensional part of the Chow ring of $X$. We proceed as in [E-P-S] and we consider the following trivial bundles on $U$ : $E_{U}:=E \times U$ and $F_{U}:=F \times U$. The group $G_{1}$ acts on $E_{U}$ in the following way: $(\alpha, \beta, \gamma)(\mathbf{e}, f, \mathbf{v})=\left(\beta(\mathbf{e}),\left(\beta \otimes i d_{V}\right) \circ f \circ \alpha^{-1}+\left(\beta \circ \gamma \circ \alpha^{-1}\right)_{\mathbf{v}}, \mathbf{v}\right)$ for any $\mathbf{e} \in E$ and $(f, \mathbf{v}) \in U$. The group $G_{1}$ acts similarly on $F_{U}$.

If we fix an element $\boldsymbol{\lambda} \in \operatorname{Hom}\left(\Lambda^{3}(E), \Lambda^{2}(F)\right)$ we also get an action of $G$ on $E_{U}^{\prime}:=E_{U} \otimes\langle\boldsymbol{\lambda}\rangle=(E \otimes\langle\boldsymbol{\lambda}\rangle) \times U$ in this way: $(\alpha, \beta, \gamma)(\mathbf{e} \otimes \tau \boldsymbol{\lambda}, f, \mathbf{v})=$ $\left(\beta(\mathbf{e}) \otimes \tau\left(\frac{\operatorname{det}(\alpha)}{\operatorname{det}(\beta)}\right) \boldsymbol{\lambda},\left(\beta \otimes i d_{V}\right) \circ f \circ \alpha^{-1}+\left(\beta \circ \gamma \circ \alpha^{-1}\right)_{\mathbf{v}}, \mathbf{v}\right)$ where $\tau \in \mathbb{C}$. Note that the action of $\Gamma$ on $E_{U}^{\prime}$ is trivial so we get a true action of $G$.

Note also that the action of $G$ commutes with the natural projection $E_{U}^{\prime} \rightarrow U$. Similarly we have an action of $G$ on $F_{U}^{\prime}:=F_{U} \otimes<\boldsymbol{\lambda}>=(F \otimes<\boldsymbol{\lambda}>) \times U$. It is necessary to introduce $E_{U}^{\prime}$ and $F_{U}^{\prime}$ because, in general, $G$ does not act on $E_{U}$ and $F_{U}$. 
Now $E_{U}^{\prime}$ and $F_{U}^{\prime}$ are $G$-bundles over $U$ and they give rise to two vector bundles $\mathcal{E}$ and $\mathcal{F}$ over $X$ as $G$ acts freely on $U$.

We can argue as in the proof of Proposition 2 of [E-P-S] with the only difference that here $U$ is not affine. However $U$ is fibered over $\mathbb{P}^{4}(\mathbb{C})^{*}$ by the restriction of the natural map $p: W \rightarrow \mathbb{P}^{4}(\mathbb{C})^{*}$. By abuse of notation let us call $p$ its restriction to $U$. The fibres of $p$ are open subsets of $\mathbb{A}^{24}(\mathbb{C})$. Thus, by 1.9 .2 of $[\mathrm{F}]$, we have that $p^{*}: A_{k}\left(\mathbb{P}^{4}(\mathbb{C})^{*}\right) \rightarrow A_{k+24}(U)$ is surjective $\forall k \geq 0$. In particular $p^{*}: A_{3}\left(\mathbb{P}^{4}(\mathbb{C})^{*}\right)=$ $A^{1}\left(\mathbb{P}^{4}(\mathbb{C})^{*}\right):=<H>\rightarrow A_{27}(U)=A^{1}(U)$ is surjective.

Let us consider the vector bundle $\mathcal{E} \oplus \mathcal{F}$ over $X$ whose structure group is $G_{2}:=$ $G L(E) \times G L(F)$. Let $T$ be the principal $G_{2}$-homogeneous bundle $\varphi: T \rightarrow X$ associated to $\mathcal{E} \oplus \mathcal{F}$. As in [E-P-S] (see also Remarque p.4-35 of [C]) we have that $A(T) \simeq A(X) /\left(c_{i}(\mathcal{E}), c_{i}(\mathcal{F})\right)$, where $\left(c_{i}(\mathcal{E}), c_{i}(\mathcal{F})\right)$ is the ideal generated by the Chern classes of $\mathcal{E}$ and $\mathcal{F}$, and $c_{1}(\mathcal{E})=c_{1}(\mathcal{F})$. Let $\tau$ be the class of the cycle $c_{1}(\mathcal{E})=c_{1}(\mathcal{F})$ in $A(X)$. Note that $\varphi$ factors through: $T \rightarrow U \rightarrow X$, because $\varphi^{*} \mathcal{E}$ and $\varphi^{*} \mathcal{F}$ are trivial, and moreover the map $T \rightarrow U$ is a $\mathbb{C}^{*}$-bundle. Hence the induced map: $A^{1}(U) \rightarrow A^{1}(T)$ is surjective and, by recalling the properties of $p^{*}$, we have a surjective map: $\langle H\rangle \rightarrow A^{1}(T)$. Therefore $A^{1}(X)=\langle\tau, \eta\rangle$, where $\eta$ is the image class of $H$ and we get $i i i)$ of 2.1 .

Note that the generator $\tau$ of $A^{1}(X)$ cuts every fibre $X_{\mathbf{v}}$ along the unique numerical class of divisors of the compactified space of twisted cubics in $\mathbb{P}^{3}(\mathbb{C})$. It suffices to remark that $X \subset \mathcal{G}$ is fibered on $\mathbb{P}^{4}(\mathbb{C})^{*}$ in such a way that the fibres are [E-P-S] compactifications of the spaces of twisted cubics in the hyperplanes of $\mathbb{P}^{4}(\mathbb{C}) . \tau$ cuts every fibre along the single generator of the codimension 1 part of the Chow ring of these compactificatons. By [E-P-S] we know that the complements of such generators are exactly the spaces of the twisted cubics in the hyperplanes. It follows that the complement of $\tau$ in $X$ is the space of the twisted cubics in $\mathbb{P}^{4}(\mathbb{C})$. Moreover we also get: $\tau^{13}=0$ because the dimension of any fibre $X_{\mathbf{v}}$ is 12 .

Now we want to consider another system of generators for $A^{1}(X) \otimes \mathbb{Q}$. The cycle consisting of all cubics in $\mathbb{P}^{4}$ intersecting a given plane in $\mathbb{P}^{4}$ represents a class in $A^{1}(X) \otimes \mathbb{Q}$, (see $[\mathrm{E}-\mathrm{S}]$ p.31) denoted by $A$. Note that $\eta$ is the class of the cycle consisting of all cubics belonging to any of the hyperplanes passing through a fixed point in $\mathbb{P}^{4}$. Since $\operatorname{dim}\left[A^{1}(X) \otimes \mathbb{Q}\right]=2$, then $A$ and $\eta$ are generators of $A^{1}(X) \otimes \mathbb{Q}$; hence $\tau=x A+y \eta$ where $x, y \in \mathbb{Q}$. Let us calculate $x$ and $y$.

Proposition 2.6. With the previous notation, $\tau=2 A-3 \eta$.

Proof. Let $\Sigma$ be the cubic surface in $\mathbb{P}^{4}$ which is a rational ruled surface $\mathbb{F}_{1}$ embedded by avery ample line bundle numerically equivalent to $C_{0}+2 f$ (see $[\mathrm{H}], \mathrm{V} .2$ ) and let us consider a generic pencil of hyperplanes in $\mathbb{P}^{4}$. The hyperplanes of the pencil give rise to a rational curve $\Gamma_{1}$ in $X$ such that $\Gamma_{1} \tau=3$, because there are exactly 3 points on $\Gamma_{1}$ corresponding to curves that are not twisted cubics: they are the curves that are sections with tangent hyperplanes, and they are 3 as the class of $\Sigma$ is 3 (see $[\mathrm{F}]$, Ex. 14.4.5). Moreover: $\Gamma_{1} A=3$, because any generic plane in $\mathbb{P}^{4}$ cuts $\Sigma$ at $3=\operatorname{deg}(\Sigma)$ distinct points and there is only one curve of the family passing through anyone of these points, and $\Gamma_{1} \eta=1$, because for any generic point in $\mathbb{P}^{4}$ there passes only one hyperplane of the pencil.

Hence we have: $3=3 x+y$.

Now let $X_{\mathbf{v}}$ be a generic fibre of $X$ and let us restrict $\tau, A, \eta$ to it. Denote by $\tau^{\prime}$ and $A^{\prime}$ the restrictions of $\tau$ and $A$ to $X_{\mathbf{v}}$, (clearly $X_{\mathbf{v}} \eta=0$ ). We have $\tau^{\prime}=x A^{\prime}$ and 
we can consider $\tau^{\prime}$ as the class of the cycle of the degenerate cubic curves in a fixed projective 3 -space $P$ and $A^{\prime}$ as the class of the cycle of twisted cubics intersecting a given line in $P$ (see [E-S] pag. 31). To calculate $x$ we use a suitable curve $\Gamma_{2}$ in $X_{\mathbf{v}}$. Let $a, h, c, d, e, f$ six generic linear forms in $P$. The following quadrics:

$a h+c d=0, \lambda e h+\mu f d=0, \lambda e c-\mu f a=0$,

depending on two projective parameters $(\lambda: \mu)$, give rise to a rational family of cubic curves, all lying on the quadric $a h+c d=0$, i.e. to a rational cubic curve $\Gamma_{2}$ in $X_{\mathbf{v}}$, such that: $\Gamma_{2} \tau^{\prime}=4$.

In fact $\Gamma_{2} \tau^{\prime}$ is the number of reducible cubic curves (conic+line) belonging to the family and such curves are necessarily composed by a line lying on the smooth quadric $a h+c d=0$; it is easy to see that there are only 4 such cubics, two of them for $(1: 0)$ and $(0: 1)$.

Moreover $\Gamma_{2} A^{\prime}=2$, because a generic line in $P$ cuts the first quadric on two points and there is only one cubic of the family passing through each one of these two points.

Hence $x=2$ and therefore $y=-3$.

Now let us consider a surface $S$ as it was defined in $\S 1$. Let $\Gamma$ be the corresponding curve in $X$. From Proposition 2.6 it follows that $\Gamma \tau=\Gamma(2 A-3 \eta)=2 \Gamma A-3 \Gamma \eta$. hence the number of singular fibre of $S$ can be calculated by $\Gamma A$ and $\Gamma \eta$. Moreover we have that $\Gamma A=\operatorname{deg}(S)$ because any generic plane in $\mathbb{P}^{4}$ cuts $S$ at $\operatorname{deg}(S)$ points and there is only one cubic of the ruling passing through each of these points. On the other hand, $\Gamma \eta$ is the number of cubics of $\Gamma$ belonging to the hyperplanes passing through a fixed generic point of $\mathbb{P}^{4}$; i.e. the number of plane nodal cubics occurring in a generic projection of $S$ into $\mathbb{P}^{3}$; i.e. the degree of the curve in $\mathbb{P}^{4 *}$ corresponding to the hyperplanes spanned by the cubics.

Remark 2.1. Using the ideas developed in this section it is also possible to show that there exists a minimal compactification $X^{\prime}$ of the space of twisted cubics in $\mathbb{P}^{r}(\mathbb{C}), r \geq 4$, such that

i) $X^{\prime}$ is a smooth projective variety of dimension $12+4(r-3)$.

ii) $X^{\prime}$ is a fibered space over $G(3, r)$, and every fibre is isomorphic to a suitable compactification of the space of twisted cubics in $\mathbb{P}^{3}(\mathbb{C})$.

iii) $A^{1}\left(X^{\prime}\right) \otimes \mathbb{Q}$ is generated by two elements, say $\eta$ and $\tau$, in such a way that $\eta$ is the pull-back of the generator of $A^{1}(G(3, r))$ and the complement of $\tau$ in $X^{\prime}$ is the space of the twisted cubics in $\mathbb{P}^{r}(\mathbb{C})$.

\section{Some Rational RUled surfaces}

In [E] the author considers smooth, rational, non degenerate, surfaces $S$ in $\mathbb{P}^{4}$ ruled in cubics or quartics (i.e. possessing a base point free pencil of rational curves of degree 3 or 4 in $\mathbb{P}^{4}$ ) and he shows that $\operatorname{deg}(S) \leq 12$. Let $d$ be the degree of $S$ and let $g$ be its sectional genus. In particular, when $S$ is ruled in cubics, in prop. 5 of $[\mathrm{E}]$, it is proved that the only possibilities for $(d, g)$ are: $(5,2),(6,3),(7,5),(8,8),(9,12)$.

In this section $S$ is always a surface of the previous type.

Let $\mathbb{F}_{e}=\mathbb{P}\left(\mathcal{O}_{\mathbb{P}^{1}} \oplus \mathcal{O}_{\mathbb{P}^{1}}(-e)\right)$ be the rational ruled surface of invariant $e \geq 0$. $\operatorname{Num}\left(\mathbb{F}_{e}\right)=\left\langle C_{0}, f\right\rangle$, where $f$ is the numerical class of any fibre of $\mathbb{F}_{e}$ and $\bar{C}_{0}$ is the numerical class of the fundamental section. Clearly every previously considered surface $S$ is the blow up, at some 0 -dimensional subscheme, of some $\mathbb{F}_{e}$, embedded by a very ample divisor $L \equiv \sigma^{*}\left(3 C_{0}+b f\right)-\mathcal{E}$, where $\sigma: S \rightarrow \mathbb{F}_{e}$ is the blow up, 
$\mathcal{E}$ is the effective exceptional divisor and $\equiv$ means numerical equivalence. In this section we will prove the following.

Theorem 3.1. Let $S$ be a surface as above. If $d \geq 7, S$ does not exist. If $d=6$, $S$ is $\mathbb{F}_{1}$ embedded by $L \equiv \sigma^{*}\left(3 C_{0}+4 f\right)-\sum_{i=1}^{9} \mathcal{E}_{i}$ (blow up at 9 points in general position). If $d=5, S$ is $\mathbb{F}_{1}$ embedded by $L \equiv \sigma^{*}\left(3 C_{0}+4 f\right)-\sum_{i=1}^{6} \mathcal{E}_{i}-2 \mathcal{E}_{7}$ (blow up at 7 points in general position).

First of all we need the following lemma.

Lemma 3.2. Let $\mathbb{F}_{e}$ be the rational ruled surface of invariant $e \geq 0$. Let $\sigma: \Sigma \rightarrow \mathbb{F}_{e}$ be a blow up of $\mathbb{F}_{e}$ at some 0-dimensional subscheme. Let $L$ be a very ample divisor on $\Sigma$. Let us assume that $L$ embeds $\Sigma$ in such a way that the fibres of $\mathbb{F}_{e}$ are mapped into the elements of a base point free pencil of rational curves of degree 3 , hence $L$ $\equiv \sigma^{*}\left(3 C_{0}+b f\right)-\mathcal{E}$ for some suitable integer $b$ and for some effective exceptional divisor $\mathcal{E}$. Then:

$i)$ the above effective exceptional divisor $\mathcal{E}$ is of the following type:

$$
\mathcal{E}=\sum_{i=1}^{h} \mathcal{E}_{i}+\sum_{j=1}^{k} 2 \mathcal{E}_{j}+\sum_{p=1}^{l}\left(\mathcal{E}_{p}^{\prime}+\mathcal{E}_{p}^{\prime \prime}\right)+\sum_{q=1}^{m}\left(2 \mathcal{E}_{q}^{\prime}+\mathcal{E}_{q}^{\prime \prime}\right)
$$

where all effective divisors $\mathcal{E}_{*}$ are disjoint and with selfintersection -1 ;

ii) the fibres not embedded as twisted cubics are embedded as: smooth conics and a line intersecting transversally the conic at one point; or: singular conics, having only one singular point, and a line intersecting tranversally the conic at a smooth point.

Proof. It is well known that the elements of the very ample linear system which embeds $\Sigma$ are in one to one correspondence with the elements of some linear system $|D|$ on $\mathbb{F}_{e}$ passing through the 0 -dimensional subscheme $\Delta$ we blow up to get $\Sigma$, where $D$ is an effective divisor on $\mathbb{F}_{e}$. Moreover $D \equiv 3 C_{0}+b f$ because every fibre has to be sent into a cubic curve.

Let us fix a fibre $\bar{f}$ of $\mathbb{F}_{e}$ and let us consider all possible components of $\Delta$ belonging to it. We proceed in 5 steps.

1) Let us suppose that there is only one simple point $P$ of $\Delta$ on $\bar{f}$. Let $\sigma_{1}$ : $\Sigma_{1} \rightarrow \mathbb{F}_{e}$ be the blow up of $\mathbb{F}_{e}$ at $P$. We get an exceptional divisor $E_{1}$ and the fibre corresponding to $\bar{f}$ is given by two components: $\sigma_{1}^{*} \bar{f}-E_{1}$ and $E_{1}$ intersecting tranversally at one point. We have $\left(\sigma_{1}^{*} D-E_{1}\right)\left(\sigma_{1}^{*} \bar{f}-E_{1}\right)=2$ and $\left(\sigma_{1}^{*} D-E_{1}\right) E_{1}=1$. If the exceptional divisor $\mathcal{E}_{i}$ corresponding to $E_{1}$, appears with coefficient -1 in the numerical class of $L$, the component $\sigma_{1}^{*} \bar{f}-E_{1}$ is embedded as a conic and $E_{1}$ is embedded as a line. If the exceptional divisor $\mathcal{E}_{j}$ corresponding to $E_{1}$ appears with coefficient -2 in the numerical class of $L$, the component $\sigma_{1}^{*} \bar{f}-E_{1}$ is embedded as a line and $E_{1}$ is embedded as a conic. Note that the coefficient can not be $\leq-3$, otherwise every element of $|L|=\left|\sigma^{*} D-\mathcal{E}\right|$ passing through any generic point of $\bar{f}$ would contain $\bar{f}$ and therefore $\sigma^{*} D-\mathcal{E}$ could not be very ample. If we assume that there are $h$ points of the first type and $k$ points of the second type we get the first part of (1).

2) Now let us suppose that there are only two single simple points $P^{\prime}$ and $P^{\prime \prime}$ of $\Delta$ on $\bar{f}$. Let $\sigma_{1}: \Sigma_{1} \rightarrow \mathbb{F}_{e}$ be the blow up of $\mathbb{F}_{e}$ at $P^{\prime}$ and $P^{\prime \prime}$. We get two exceptional divisor $E_{1}^{\prime}$ and $E_{1}^{\prime \prime}$ and the fibre corresponding to $\bar{f}$ is given by three 
components: $\sigma_{1}^{*} \bar{f}-E_{1}^{\prime}-E_{1}^{\prime \prime}, E_{1}^{\prime}$ and $E_{1}^{\prime \prime}$ intersecting piarwise tranversally at one point. We have $\left(\sigma_{1}^{*} D-E_{1}^{\prime}-E_{1}^{\prime \prime}\right)\left(\sigma_{1}^{*} \bar{f}-E_{1}^{\prime}-E_{1}^{\prime \prime}\right)=1,\left(\sigma_{1}^{*} D-E_{1}^{\prime}-E_{1}^{\prime \prime}\right) E_{1}^{\prime}=1$, $\left(\sigma_{1}^{*} D-E_{1}^{\prime}-E_{1}^{\prime \prime}\right) E_{1}^{\prime \prime}=1$. The exceptional divisors $\mathcal{E}_{p}^{\prime}$ and $\mathcal{E}_{p}^{\prime \prime}$ corresponding to $E_{1}^{\prime}$ and $E_{1}^{\prime \prime}$ appear with coefficient -1 in the numerical class of $L$ and every component is embedded as a line. Note that the coefficents can not be $\leq-2$ for the same argument as above. If we assume that there are $l$ pairs of points of this type we get the third summand of (1).

3) As every fibre $\bar{f}$ of $\mathbb{F}_{e}$ is tranformed by the blow up into a curve which has to be embedded as a cubic by $|L|$, it can not have more than 3 irreducible components, so that on $\bar{f}$ can not be more than two distinct simple points of $\Delta$. Now let us consider non reduced points of $\Delta$. Let us assume that there is only one double point of $\Delta$ on $\bar{f}$. We can consider it as a couple $(P, v)$ where $v \in T_{P}\left(\mathbb{F}_{e}\right)$ the analytic tangent space of $\mathbb{F}_{e}$ at $P$. Firstly let us assume that $v \notin T_{P}(\bar{f})$. In this case let $\sigma_{1}: \Sigma_{1} \rightarrow \mathbb{F}_{e}$ be the blow up of $\mathbb{F}_{e}$ at $P$. We get one exceptional divisor $E_{1}$ and the fibre corresponding to $\bar{f}$ is given by two components: $\sigma_{1}^{*} \bar{f}-E_{1}$ and $E_{1}$ intersecting tranversally at one point. Let $\sigma_{2}: \Sigma_{2} \rightarrow \Sigma_{1}$ be the blow up of $\Sigma_{1}$ at the point of $E_{1}$ corresponding to $v$. We get another exceptional divisor $E_{2}$ and the fibre corresponding to $\bar{f}$ is given by three components: $\sigma_{2}^{*}\left(\sigma_{1}^{*} \bar{f}-E_{1}\right), \sigma_{2}^{*} E_{1}-E_{2}$ and $E_{2}$, two by two intersecting transversally at one point only. We have:

$$
\begin{aligned}
& {\left[\sigma_{2}^{*}\left(\sigma_{1}^{*} D\right)-\sigma_{2}^{*} E_{1}-E_{2}\right]\left[\sigma_{2}^{*}\left(\sigma_{1}^{*} \bar{f}-E_{1}\right)\right]=\left(\sigma_{1}^{*} D-E_{1}\right)\left(\sigma_{1}^{*} \bar{f}-E_{1}\right)=2} \\
& {\left[\sigma_{2}^{*}\left(\sigma_{1}^{*} D\right)-\sigma_{2}^{*} E_{1}-E_{2}\right]\left(\sigma_{2}^{*} E_{1}-E_{2}\right)=\left(\sigma_{1}^{*} D-E_{1}\right) E_{1}-1=0} \\
& {\left[\sigma_{2}^{*}\left(\sigma_{1}^{*} D\right)-\sigma_{2}^{*} E_{1}-E_{2}\right] E_{2}=1 .}
\end{aligned}
$$

It is easy to see that the only possibility to have $L$ very ample is that the exceptional divisors $\mathcal{E}_{q}^{\prime}$ corresponding to $\sigma_{2}^{*} E_{1}$ appears with coefficient -2 in the numerical class of $L$ while the exceptional divisors $\mathcal{E}_{q}^{\prime \prime}$ corresponding to $E_{2}$ appears with coefficient -1 ; in this case every component is embedded as a line. If there are $m$ points of this type we get the last part of (1).

4) Now let us assume that there is only one double point of $\Delta$ on $\bar{f}$ such that it can be considered as a couple $(P, v)$ with $v \in T_{P}(\bar{f})$. In this case let $\sigma_{1}: \Sigma_{1} \rightarrow \mathbb{F}_{e}$ be the blow up of $\mathbb{F}_{e}$ at $P$. We get one exceptional divisor $E_{1}$ and the fibre corresponding to $\bar{f}$ is given by two components: $\sigma_{1}^{*} \bar{f}-E_{1}$ and $E_{1}$ intersecting tranversally at one point $Q$. Let $\sigma_{2}: \Sigma_{2} \rightarrow \Sigma_{1}$ be the blow up of $\Sigma_{1}$, necessarily at $Q$. We get another exceptional divisor $E_{2}$ and now the fibre corresponding to $\bar{f}$ is given by these three components: $\sigma_{2}^{*}\left(\sigma_{1}^{*} \bar{f}-E_{1}\right)-E_{2}, \sigma_{2}^{*} E_{1}-E_{2}$ and $E_{2}$, two by two intersecting transversally at one point only. We have:

$$
\begin{aligned}
& {\left[\sigma_{2}^{*}\left(\sigma_{1}^{*} D\right)-\sigma_{2}^{*} E_{1}-E_{2}\right]\left[\sigma_{2}^{*}\left(\sigma_{1}^{*} \bar{f}-E_{1}\right)-E_{2}\right]=\left(\sigma_{1}^{*} D-E_{1}\right)\left(\sigma_{1}^{*} \bar{f}-E_{1}\right)-1=1} \\
& {\left[\sigma_{2}^{*}\left(\sigma_{1}^{*} D\right)-\sigma_{2}^{*} E_{1}-E_{2}\right]\left(\sigma_{2}^{*} E_{1}-E_{2}\right)=\left(\sigma_{1}^{*} D-E_{1}\right) E_{1}-1=0} \\
& {\left[\sigma_{2}^{*}\left(\sigma_{1}^{*} D\right)-\sigma_{2}^{*} E_{1}-E_{2}\right] E_{2}=1 .}
\end{aligned}
$$

It is easy to see that it is not possible that there exist exceptional divisors $\mathcal{E}_{s}^{\prime}$, corresponding to $\sigma_{2}^{*} E_{1}$, and $\mathcal{E}_{s}^{\prime \prime}$, corresponding to $E_{2}$, in the numerical class of $L$ in such a way that every component of the fibre corresponding to $\bar{f}$ is embedded as a line. Hence this case can not be possible if $L$ is very ample.

5 ) It is not possible that on $\bar{f}$ there are two or more double points of $\Delta$, otherwise the corresponding fibre would have more than 3 irreducible components and this is not possible as it must be embedded as a cubic curve. For the same reason it is not possible that on $\bar{f}$ there are one or more multiple points of $\Delta$. Hence our analysis is complete and we have proved $i$ ). Moreover, along the way, we have also proved ii). 
Now let us reconsider a surface $S$. We have the following lemmas.

Lemma 3.3. Let $S \subset \mathbb{P}^{4}$ be a surface as above. We know that $S$ is the blow up of some $\mathbb{F}_{e}$ at some 0 -dimensional scheme and it is embedded in $\mathbb{P}^{4}$ by a very ample divisor $L \equiv \sigma^{*}\left(3 C_{0}+b f\right)-\mathcal{E}$ as in lemma 3.2. Then, according to the possible degrees of $S: 5,6,7,8,9$, the sum $h+k+2 m+2 l$ is: $7,9,15,25,39$, respectively. Moreover $2 d \geq h+k+m+l$.

Proof. By lemma 3.2 we know that

$$
K_{S} \equiv \sigma^{*}\left(-2 C_{0}-(e+2) f\right)+\sum_{i=1}^{h} E_{i}+\sum_{j=1}^{k} E_{j}+\sum_{p=1}^{l}\left(E_{p}^{\prime}+E_{p}^{\prime \prime}\right)+\sum_{q=1}^{m}\left(E_{q}^{\prime}+E_{q}^{\prime \prime}\right)
$$

hence $K_{S}^{2}=8-(h+k+2 l+2 m)$. On the other hand, as $S$ is embedded as a smooth (non degenerated) surface in $\mathbb{P}^{4}$, we have the well known condition (see $[\mathrm{H}]$ pag. 434 , the following version is concerning rational ruled surfaces):

$$
d(d-5)-10(g-1)=2 K_{S}^{2}-12
$$

implying: $h+k+2 l+2 m=-\frac{d(d-5)}{2}+5(g-1)+2$.

For the 5 types of surfaces $S$ under consideration we have:

$(5,2) ; h+k+2 l+2 m=7$

$(6,3) ; h+k+2 l+2 m=9$

$(7,5) ; h+k+2 l+2 m=15$

$(8,8) ; h+k+2 l+2 m=25$

$(9,12) ; h+k+2 l+2 m=39$.

Now, as in $\S 1$, we can consider the curve $\Gamma$ in $X$ arising from the surface $S$.

Obviously we have $\Gamma \tau \geq h+k+l+m$ (equality holds when the intersection is transverse) and, by using lemma 2.6, we have that:

$$
0 \leq 3 \Gamma \eta=\Gamma(2 A-\tau)=2 d-\Gamma \tau .
$$

From $\left(^{\wedge}\right)$ we conclude our proof.

Lemma 3.4. Let $S \subset \mathbb{P}^{4}$ be a surface as above, having $(d, g)=(5,2),(6,3),(7,5)$, $(8,8),(9,12)$. Then: $h+k+m+l=3 g+6-d$.

Proof. Let us project $S$ into some generic plane $\Pi \simeq \mathbb{P}^{2}$ by a generic line $\lambda$ in $\mathbb{P}^{4}$. We get a one dimensional family $\mathcal{C}$ of nodal plane curves whose generic member is a plane cubic with only one node. In $\mathcal{C}$ there are exactly $h+k$ elements reducible into a smooth conic and a trasverse secant line and exactly $l+m$ elements reducible into 3 distinct, non collinear, lines. Note that every reduced cubic of these types has only one distinguished double point coming from the projection, while the others come from singular points on the curves in $\mathbb{P}^{4}$.

For any family as $\mathcal{C}$, such that any member is a nodal cubic with the exception of a finite number of cuspidal cubics and a finite number $\psi$ of cubics reducible into a smooth conic and a secant line, in $[\mathrm{K}-\mathrm{S}]$ it is proved the following relation (due to Zeuthen):

$$
3 \mu^{\prime}=2 \psi+2 \mu
$$

where $\mu$ is the number of elements of $\mathcal{C}$ passing through a generic point of $\mathbb{P}^{2}$ and $\mu^{\prime}$ is the number of elements of $\mathcal{C}$ tangent to a generic line in $\mathbb{P}^{2}$. 
In fact, in our case, $\mathcal{C}$ has also a finite number of cubics reducible into 3 distinct non collinear lines, however (2) can be applied because it follows from a corresponding relation among numerical classes of divisors in the space $\mathbf{N}$ of the nodal plane cubics (see $[\mathrm{K}-\mathrm{S}]$, theorem 4.9 ) which are cut by $\mathcal{C}$. The stratification of $\mathbf{N}$, under the action of $P G L(2, \mathbb{C})$, tell us that the set of the cubics reducible into 3 distinct non collinear lines with a distiguished singular point (as in our case) is a suborbit of the one-codimensional orbit of cubics reducible into a smooth conic and a secant line (with a distiguished singular point, as in our case) (see [M-X]). So that, in our case, simply $\mathcal{C}$ intersects the orbit of cubics reducible into a smooth conic and a secant line at some points belonging to that suborbit.

Let us consider $\mu$ and $\mu^{\prime}$. It is easy to see that, for a generic line $\lambda, \mu$ is equal to the degree $d$ of $S$. On the other hand, for any generic line in $\Pi$, let us consider the hyperplane $\Lambda$ of $\mathbb{P}^{4}$ spanned by this line and $\lambda$ and the smooth, genus $g$, hyperplane section $\Lambda \cap S$. There is a natural $(3: 1)$ covering $\pi_{\mid \Lambda \cap S}: \Lambda \cap S \rightarrow C \simeq \mathbb{P}^{1}$ induced by the restriction of $\pi$ and $\mu^{\prime}$ is exactly the order of the branching divisor of this covering, i.e. $\mu^{\prime}=2 g+4$. By using (2) we have: $h+k+l+m=3 g+6-d$.

Now we can put all thing together.

Proof. (of theorem 3.1). Let us examine our 5 cases.

Case $(9,12)$. By lemma 3.4 we have: $h+k+l+m=33$, but this is not possible by $\left({ }^{\wedge}\right)$, so that the surface does not exist.

Case $(8,8)$. By lemma 3.4 we have: $h+k+l+m=22$, but this is not possible by $\left({ }^{\wedge}\right)$, so that the surface does not exist.

Case $(7,5)$. By lemma 3.3 we have: $h+k+2 l+2 m=15$ and by lemma 3.4 we have: $h+k+l+m=14$, so that $l+m=1$ and $h+k=13$; ( $\left.{ }^{\wedge}\right)$ does not give contradictions but if we calculate $L^{2}=-9 e+6 b-3 k-3 m-15=d=7$, i.e. $3(-3 e+2 b-k-m-5)=7$ we see that this is not possible, so that the surface does not exist.

Case $(6,3)$. By lemma 3.3 we have: $h+k+2 l+2 m=9$ and by lemma 3.4 we have: $h+k+l+m=9$, so that $l=m=0$ and $h+k=9$; ( $\left.{ }^{\wedge}\right)$ does not give contradictions. $S$ is not necessarily a linearly normal surface, but, in any case, it is the smooth projection of a linearly normal surface of degree 6 and genus 3 , so that we can look at the list of such surfaces contained in $[\mathrm{I}]$. It is very easy to see that there is only one possibility: the blow up of $\mathbb{P}^{2}$ at 10 points in general position embedded by the pull back of the linear systems of quartics passing through these points (Bordiga surface). As $\mathbb{F}_{1}$ is the blow up of $\mathbb{P}^{2}$ at one point, we have that in this case $S$ do exist, it is linearly normal, it is the blow up of $\mathbb{F}_{1}$ at 9 points in general position and the very ample line bundle embedding $S$ in $\mathbb{P}^{4}$ is $L \equiv \sigma^{*}\left(3 C_{0}+4 f\right)-\sum_{i=1}^{9} \mathcal{E}_{i}$ $(h=9, k=0)$.

Case $(5,2)$. By lemma 3.3 we have: $h+k+2 l+2 m=7$ and by lemma 3.4 we have: $h+k+l+m=7$, so that $l=m=0$ and $h+k=7$; (^) does not give contradictions. By arguing as in the previous case we get that $S$ do exist, it is linearly normal, it is the blow up of $\mathbb{F}_{1}$ at 7 points in general position and the very ample line bundle embedding $S$ in $\mathbb{P}^{4}$ is $L \equiv \sigma^{*}\left(3 C_{0}+4 f\right)-\sum_{i=1}^{6} \mathcal{E}_{i}-2 \mathcal{E}_{7}(h=6, k=1)$. 


\section{The CASE OF $\mathbb{P}^{1}$-BUndLES.}

Let $S$ be a smooth, nondegenerate, projective surface in $\mathbb{P}^{4}(\mathbb{C})$. Let $d$ be the degree of $S$. Let us assume that $S=\mathbb{P}(E)$, where $E$ is a normalized rank 2 vector bundle, of invariant $e:=-\operatorname{deg}\left[c_{1}(E)\right]$, over a smooth curve $C$ of genus $g$ (see $\left.[\mathrm{H}]\right)$. Let $L$ be the very ample divisor $\mathcal{O}_{S}(1)$. It is well known that $L \equiv a C_{0}+b f$, (notation as in $\S 3)$.

What are the effective values of $a$ and $b$ for which such a surface $S$ do exists ? Up to now we have no answer for this simple question. In this paragraph we summarize the state of the art, after Holme and Roberts (see $[\mathrm{H}-\mathrm{R}]$ ). Note that $a$, necessarily a positive integer as $L$ is very ample, is the degree of rational curves corresponding to fibres of the embedding.

Let us start by considering the case $a=1$. It is well known that the only surface scrolls contained in $\mathbb{P}^{4}$ are the rational cubic scroll $(a=1, b=2, e=1, g=0, d=3$ ) and the elliptic quintic scroll $(a=1, b=2, e=-1, g=1, d=5)$ (see [A2] and [L]). Obviously the quadric surface is not considered here because it is degenerate. In the case $a=2$, it is known that there are no $\mathbb{P}^{1}$-bundles in $\mathbb{P}^{4}$ that are conic bundles over a curve, see $[\mathrm{E}-\mathrm{Sa}]$ and $[\mathrm{B}-\mathrm{R}]$, see also $[\mathrm{A}-\mathrm{D}-\mathrm{S}]$ for the complete classification of all conic bundles in $\mathbb{P}^{4}$. So we can assume $a \geq 3$.

Now let us consider the possible values of $g$. It is easy to see that, for $g=0$, the unique existing surface $S$ is the cubic scroll (recall that the quadric surface is not considered). For $g=1$ we have only the quintic scroll, see $[\mathrm{L}-\mathrm{P}]$ th. 3.1. Hence we can assume $g \geq 2$. Moreover we can also assume that $e<0$ by proposition 8.4 of [H-R]. In this case we have that $y:=2 b-a e>0$ as $L$ is very ample (see $[\mathrm{H}]$ ) and th. 8.9 of $[\mathrm{H}-\mathrm{R}]$ tells us that $a, y, g$ must satisfy the following relation:

$$
a^{2} y^{2}-(10 a-5) y-(10 a-4)(g-1)=0
$$

By looking at the proof of th. 8.9 of $[\mathrm{H}-\mathrm{R}]$ we see that there are no surfaces $S$ when $g \geq 2$ and $y=1$. By combining corollary 8.10 and lemma 8.11 of $[\mathrm{H}-\mathrm{R}]$ we also have that there are no surfaces $S$ when $g \geq 2$ and $y=2,3,4,5,6$ with only one possible exception $S_{1}$ with: $a=7, b=-6, e=-2, g=2, d=14$.

To consider the remaining cases: $a \geq 3, g \geq 2, y \geq 7$ we can compute all the possible solutions of (3), keeping in mind that $d=a y \leq 52$ because $S$ is a surface of non-general type. This fact follows from [D-S]; actually, in this paper, the proof of this bound contains a little gap, however the gap was fixed by Amasaki in [A1] (for a fuller account of the whole story see the introduction of $[\mathrm{A}-\mathrm{T}]$ ).

Now, a straightforward calculation shows that there are only two possible surfaces $S_{2}$ and $S_{3}$, of degree respectively $30(g=26)$ and $39(g=47)$, both with $a=3$. This means that every fibre of these surfaces is embedded in $\mathbb{P}^{4}$ as a twisted cubic, hence every surface gives rise to a curve $\Gamma$ in $X$ such that $\tau \Gamma=0$. By the results proved in $\S 1$ we can say that $0=\Gamma(2 A-3 \eta)=2 d-3 \Gamma \eta$ i.e. $3 \Gamma \eta=2 d$. For $S_{2}$ we have $\Gamma \eta=20$ and for $S_{3}$ we have $\Gamma \eta=26$. Unfortunately we have no other way to compute these numbers. Moreover, if we construct a generic projection of the two surfaces in some $\mathbb{P}^{2}$, as in $\S 3$, to get two families of nodal plane curves and we consider the known (to us) relations about the charachteristic numbers of these two families (see $[\mathrm{K}-\mathrm{S}]$ ), we do not find any contradiction. So we think that a new approach is needed to solve this problem. 


\section{REFERENCES}

[A1] M.Amasaki: Verification of the connectedness of space curve invariants for a special case. Comm. Algebra 32 (10), 3739-3744 (2004).

[A2] A.Aure: On surfaces in projective 4-space. Thesis, Oslo (1987).

[A-D-S] H.Abo-W.Decker-N.Sasakura: An elliptic conic bundle in $\mathbb{P}^{4}$ arising from a stable rank-3 vector bundle. Math. Z. 229 (4), 725-741 (1998).

[A-T] A.Alzati-A.Tortora: Connected monomial invariants. Mauscripta Math. 116, 125-133 (2005).

[B-R] R.Braun-K.Ranestad: Conic bundle in projective fourspace. Algebraic Geometry (Catania, 1993/Barcelona, 1994), Lecture Notes in Pure and Appl. Math. 200, 331-339, Dekker, New York, 1998.

[C] Seminaire Chevalley: Anneaux de Chow and applications. Paris, 1958.

[D-S] W.Decker-F.O.Schreyer: Non-general type surfaces in $\mathbb{P}^{4}$ : some remarks on bounds and constructions. J. Symbolic computation 29, 545-583 (2000).

[E] P.Ellia: A note on rational surfaces in $\mathbb{P}^{4}$. J. of Pure and applied Algebra 152, 83-88 (2000).

[E-P-S] G.Ellingsrud-R.Piene-S.A.Stromme: On the variety of nets of quadrics defining twisted cubic curves in Space Curves, Proc. 1985, Lecture Notes in Math.1266, Springer, Berlin, 1987.

[E-Sa] P.Ellia-G.Sacchiero: Smooth surfaces of $\mathbb{P}^{4}$ ruled in conics. Algebraic Geometry (Catania, 1993/Barcelona, 1994), Lecture Notes in Pure and Appl. Math. 200, 49-62, Dekker, New York, 1998.

[E-S] G.Ellingsrud-S.A.Stromme: The number of twisted cubic curves on the general quintic threefold. Math. Scand. 76, 5-34 (1995).

[F-M] J.Fogarty-D.Mumford: "Geometric Invariant theory". 2nd Erlangen Ed. Ergebnisse der Math. 34, Springer Verlag, 1982.

[F] W.Fulton: Intersection Theory. Springer Verlag, 1984.

[H] R. Hartshorne: Algebraic Geometry. Springer Verlag, 1977.

[H-R] A.Holme-J.Roberts On the embeddings of Projective Varieties. Algebraic Geometry (Sundance, UT, 1986), 118-146, Lecture Notes in Math. 1311, Springer, Berlin, 1988.

[K-S] S.Kleiman-R.Speiser: "Enumerative geometry of nodal plane cubics". Algebraic Geometry (Sundance, UT, 1986), 156-196, Lecture Notes in Math., 1311, Springer, Berlin, 1988.

[I] P.Ionescu: Embedded projective varieties of small invariants. In Proceedings of the Week of Algebraic Geometry Bucharest 1982, 142-186, Lecture Notes in Math. 1056, Springer, Berlin, 1984.

[L] A.Lanteri: On the existence of scrolls in $\mathbb{P}^{4}$. Rend. Accad. Lincei 69, 223-227 (1980).

[L-P] A.Lanteri-M.Palleschi: Osservazioni sulla rigata geometrica ellittica di $\mathbb{P}^{4}$. Istit. Lombardo Accad. Sci. Lett. Rend. A 112 (2), 223-233 (1978).

[M-X] J.M.Miret-S.Xambò Descamps: On the geometry of nodal plane cubics: the condition p. Enumerative algebraic geometry (Copenhagen, 1989), 169-187, Contemp. Math. 123, Amer. Math. Soc., Providence, RI, 1991.

[N] P.E.Newstead: Introduction to moduli problems and orbit spaces. Tata Institute, Springer Verlag, Bombay, 1982.

Dipartimento di Matematica Univ. di Milano, via C. Saldini 50 20133-Milano (Italy)

E-mail address: alberto.alzati@unimi.it

Dipartimento di Matematica Univ. di Milano, via C. Saldini 50 20133-Milano (Italy)

E-mail address: alfonso.tortora@unimi.it 